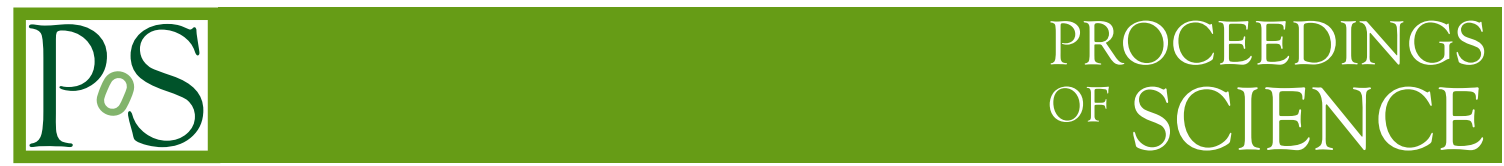

\title{
Recent measurements of the top-quark mass and Yukawa coupling using the ATLAS and CMS detectors at the LHC
}

\author{
Matteo Negrini ${ }^{\dagger}$,* \\ Istituto Nazionale di Fisica Nucleare - Sezione di Bologna, \\ Via Irnerio 46, 40126 Bologna, Italy \\ E-mail: matteo.negrini@cern.ch
}

An overview of recent measurements of the mass and Yukawa coupling of the top quark is presented. The measurements are made by the ATLAS and CMS Collaborations, exploiting $p p$ collisions at the center-of-mass energies of $\sqrt{s}=13 \mathrm{TeV}$ in the Large Hadron Collider at CERN.

The European Physical Society Conference on High Energy Physics (EPS-HEP2021), 26-30 July 2021

Online conference, jointly organized by Universität Hamburg and the research center DESY

\footnotetext{
*Speaker

$\dagger$ On behalf of the ATLAS and CMS Collaborations
} 


\section{Introduction}

In the Standard Model (SM) of particle physics, the top quark is the elementary particle with the largest mass and the largest Yukawa coupling with the Higgs boson. Its mass $\left(m_{t}\right)$ is a fundamental parameter of the SM: it allows to assess the internal consistency of the SM at the electroweak scale [1] and, assuming the validity of the SM up to very high energy scales, it has a key role in the assessment of the stability of the universe [2].

The mass of the top quark can be measured only through the comparisons of some observables, whose distributions dependend on $m_{t}$, with their theoretical expectations, and is currently measured with an uncertainty at the sub-GeV level for single measurements and reaching about half-GeV in combinations. However, the theoretical interpretation of the measured $m_{t}$ is related to the measurement technique. In "direct" measurements, $m_{t}$ is obtained from the comparison of measured distributions, such as the invariant mass of the decay products of the top quark or other quantities particularly sensitive to $m_{t}$, and their expectations obtained from Monte Carlo (MC) simulation samples generated using different $m_{t}$ values. In this way the "MC mass" $\left(m_{t}^{\mathrm{MC}}\right)$ is measured. "Indirect" measurements aim to determine $m_{t}$ from a measured cross-section that can be compared with its theoretical value, computed from first principle calculations. In this way the pole mass $\left(m_{t}^{\mathrm{POLE}}\right)$ or the mass in the modified minimal subtraction $(\overline{\mathrm{MS}})$ renormalization scheme $\left(m_{t}^{\overline{\mathrm{MS}}}\right)$, depending on the theoretical scheme that is adopted in the calculation, can be measured. The relation between $m_{t}^{\text {POLE }}$ and $m_{t}^{\overline{\mathrm{MS}}}$ is calculated to 4-loop precision in QCD [3]; the relation between $m_{t}^{\mathrm{MC}}$ and $m_{t}^{\mathrm{POLE}}$ is not well establihed and their difference, believed to be at the level of a few hundreds of MeV, is still under debate (see for example Refs. [4, 5] and references therein).

In the SM, fermions acquire their mass $m_{f}$ through a Yukawa interaction with the Higgs field, with coupling strength $g_{f}=\sqrt{2} m_{f} / v$, where $v$ is the vacuum expectation value of the Higgs field. The top quark has the largest Yukawa coupling with the Higgs boson $g_{t}^{\mathrm{SM}} \approx 1$. Possible deviations of $g_{t}$ from the expected value may indicate new physics effects that come into play to modify the effective coupling between the top quark and the Higgs field.

In the following we present an overview of some recent measurements of the mass and Yukawa coupling of the top quark, obtained by the ATLAS [6] and CMS [7] experiments, exploiting $p p$ collisions at the CERN Large Hadron Collider at the center-of-mass energies $\sqrt{s}=13 \mathrm{TeV}$.

\section{Direct top-quark mass measurements}

Jets originated by $b$ quarks can be identified by the presence of a "soft" muon inside the jet, coming from the semi-leptonic decay of a $b$-hadron. For $t \bar{t}$ events in which only one of the $t$ or $\bar{t}$ decays leptonically ${ }^{1}(l+$ jets channel), that also contain one soft-muon tagged jet, the invariant mass distribution of the pair of leptons (the soft one and the one from the leptonic top-quark decay), can be used to determine the top-quark mass. This technique has the advantage of using a fully leptonic quantity that does not require the complete reconstruction of a top-quark, therefore reducing the uncertainty related to hadronic calibration and the modeling of the top-quark kinematics. The top-quark mass measured by the ATLAS Collaboration exploiting this technique, using $36.1 \mathrm{fb}^{-1}$

\footnotetext{
1The top-quark always decays in a $b W^{+}$pair. The "leptonic" or "hadronic" top quark decay is defined depending on the subsequent $W^{+} \rightarrow l^{+} v$ or $W^{+} \rightarrow q \bar{q}^{\prime}$ decay, respectively (charge conjugation is implied in this definition).
} 
of data at $\sqrt{s}=13 \mathrm{TeV}$ [8], is $m_{t}^{\mathrm{MC}}=174.48 \pm 0.40$ (stat) \pm 0.67 (syst) $\mathrm{GeV}$, in which the main contribution to the uncertainty is due to the modelling of the $b$ fragmentation and decay.

As of today, the vast majority of $m_{t}$ measurements have been done using $t \bar{t}$ events. Thanks to the large data samples now collected by the LHC experiments, $m_{t}$ measurements using single top quark production in the $t$-channel have become competitive and useful in combinations with other measurements, that are obtained in complementary phase spaces and dominated by different sources of uncertainty. Using $35.9 \mathrm{fb}^{-1}$ of data at $\sqrt{s}=13 \mathrm{TeV}$, the CMS Collaboration obtained a sample of single top-quark events in the leptonic channel with $\sim 60 \%$ purity by means of a boosted decision tree discriminator, used to separate $t$-channel single top-quark events from backgrounds, built using variables whose correlation with $m_{t}$ is small or absent [9]. The top-quark mass is extracted by means of a maximum likelihood fit to the reconstructed $\ln m_{t}$, where the logarithm helps in the definition of appropriate parametric templates for the signal and background by reducing the skewness of the $m_{t}$ distribution. It is observed in MC simulated events that the peak of the reconstructed $m_{t}$ distribution does not correspond to the $m_{t}$ value used in the generator, mainly because a mis-reconstruction of the longitudinal momentum of the neutrino originated in the top-quark decay, therefore an "offset calibration" is applied to correct for this effect. With this techniqe, $m_{t}$ is determined in single top-quark events to be $m_{t}^{\mathrm{MC}}=172.13_{-0.77}^{+0.76} \mathrm{GeV}$, in which the uncertainty is dominated by the modeling of signal and $t \bar{t}$ events. The top quark-antiquark mass difference and mass ratio are also measured, by using the two sub-samples containing positive and negative leptons separately, to be $m_{t}-m_{\bar{t}}=0.83_{-1.01}^{+0.77} \mathrm{GeV}$ and $m_{\bar{t}} / m_{t}=0.995_{-0.006}^{+0.005}$, in line with the SM expectations.

\section{Indirect top-quark mass measurements}

The first investigation of the running of the top-quark mass in the $\overline{\mathrm{MS}}$ scheme was done by the CMS Collaboration using $35.9 \mathrm{fb}^{-1}$ of data at $\sqrt{s}=13 \mathrm{TeV}$ [10]. In this analysis, a set of $t \bar{t}$ simulated events is divided in four sub-samples, corresponding to four intervals of the invariant mass of the $t \bar{t}$ pair determined at parton-level, which are treated as independent samples at the scales $\mu_{k}(k=1,4)$ defined as the average $m_{t \bar{t}}$ in each interval. A maximum-likelihood unfolding is then applied to determine the true number of events at each scale from the observed one, which is needed to correct for reconstruction and resolution effects. For the determination of $m_{t}^{\overline{\mathrm{MS}}}$, measurements are compared with theoretical predictions at NLO in the $\overline{\mathrm{MS}}$ scheme as implemented in MCFM v6.8 [11]. The top-quark mass value at the $m_{t}$ scale, $m_{t}\left(m_{t}\right)$, is determined independently in each $m_{t \bar{t}}$ interval, and the calculation of the corresponding $m_{t}\left(\mu_{k}\right)$ is done at one loop precision using CRunDec v3.0 [12]. The measured running mass is found to agree with the expected evolution from $m_{t}\left(m_{t}\right)=162.9 \pm 1.6\left(\text { fit }+ \text { extr }+\mathrm{PDF}+\alpha_{\mathrm{S}}\right)_{-3.0}^{+2.5}($ scale $) \mathrm{GeV}$.

\section{Interpretation of the Monte Carlo top-quark mass}

The difference between $m_{t}^{\mathrm{MC}}$ and $m_{t}^{\text {pole }}$ is expected to be of the order of $0.5 \mathrm{GeV}$, due to nonperturbative QCD effects that affect the top-quark mass determination [4,5]. A study to relate $m_{t}^{\mathrm{MC}}$ to mass definitions adopted in well-defined theoretical schemes, such as $m_{t}^{\text {pole }}$ and $m_{t}^{\mathrm{MSR}}$, is done by the ATLAS Collaboration using MC simulated $t \bar{t}$ events originated from $p p$ collisions at $\sqrt{s}=13 \mathrm{TeV}$ [13]. The MSR mass scheme is similar to the $\overline{\mathrm{MS}}$ scheme but depends on an energy scale [14], which 
can be set to low values to obtain a mass value close to the pole mass: $m_{t}^{\mathrm{MSR}}(1 \mathrm{GeV}) \approx m_{t}^{\text {pole }}$. In the ATLAS study, large-radius jets originated by hadronically decaying top quarks at large transverse momenta $\left(p_{T}>750 \mathrm{GeV}\right)$, are reconstructed with the XCone algorithm with radius parameter $R=1$ and with soft radiation removed by applying the Soft-Drop algorithm with parameters $\beta=0,1,2$ and $z_{\text {cut }}=0.01,0.05$ [15]. The mass distribution of such large-R jets is compared with the distribution calculated in perturbative $\mathrm{QCD}$ at the next-to-leading-logarithmic resummation. The mass difference is found to be $\Delta m_{t}^{\mathrm{MSR}}=m_{t}^{\mathrm{MC}}-m_{t}^{\mathrm{MSR}}(1 \mathrm{GeV})=80_{-400}^{+350} \mathrm{MeV}$, in which the contributions to the uncertainty are due to theoretical calculations (obtained by varying 5 theoretical scales in the calculation: $+230 /-310 \mathrm{MeV}$ ), due to the method (obtained varying the range of large- $\mathrm{R}$ jet mass used in the comparison and the considered kinematic range: $190 \mathrm{MeV}$ ), and due to the modeling of the underlying event in the simulation $(155 \mathrm{MeV})$. The study shows that $m_{t}^{\mathrm{MC}}$ is very close to $m_{t}^{\mathrm{MSR}}(1 \mathrm{GeV})$, with an uncertainty that is confirmed to be of the order of few hundreds of $\mathrm{MeV}$.

\section{Measurement of top-quark Yukawa coupling in $t \bar{t}$ events}

The coupling of the top quark with the Higgs field $g_{t}$ has been successfully measured using the associated production of a top quark-antiquark pair with a Higgs boson $(t \bar{t} H)$. It is also possible to measure $g_{t}$ using $t \bar{t}$ events, in which the coupling with the Higgs boson enters at the loop level and can modify the shape of some kinematical distributions with respect to the SM expectations. This study has been done by the CMS Collaboration with $137 \mathrm{fb}^{-1}$ of data at $\sqrt{s}=13 \mathrm{TeV}$ [17], by reconstructing $t \bar{t}$ events in the dileptonic channel and considering the distribution of the two reconstructed quantities: the invariant mass of the system composed by the two $b$-jets and the lepton-antilepton pair, $M_{b b l l}$, and the difference in rapidity between the two $b$-jet lepton pairs, $\Delta y_{b l b l}$, in which $b$-jets and leptons are associated under the assumption that they come from the decay of the same top quark or anti-quark using kinematic constraints. The impact on the considered distributions of loop corrections for $g_{t}$ values different from 1 is estimated by reweighting simulated $t \bar{t}$ events using correction factors derived from the HATHOR package [16]. By means of a profile likelihood scan the coupling strength is measured to be $Y_{t}=g_{t} / g_{t}^{\mathrm{SM}}=1.16_{-0.08}^{+0.07}(\mathrm{stat})_{-0.34}^{+0.23}$ (syst), in excellent agreement with the SM expectations.

\section{Summary}

The mass of the top quark is a fundamental parameter of the SM. Its measurement at a precision of a few hundreds of $\mathrm{MeV}$ at the LHC poses interesting experimental and theoretical challenges. Different experimental techniques may require different theoretical interpretations, whose relations require further investigations, such as the one between $m_{t}^{\mathrm{MC}}$ and $m_{t}^{\text {pole }}$ or $m_{t}^{\overline{\mathrm{MS}}}$.

Measurement of the top-quark Yukawa coupling using $t \bar{t}$ events are potentially sensitive to new physics effects that contribute to loop corrections. A recent measurement of the coupling strength $Y_{t}=1.16_{-0.08}^{+0.07}$ (stat) ${ }_{-0.34}^{+0.23}$ (syst) shows no deviations with respect to the SM expectations. 


\section{References}

[1] M. Baak et al. [Gfitter Group], The global electroweak fit at NNLO and prospects for the LHC and ILC, Eur. Phys. J. C 74 (2014), 3046 [arXiv:1407.3792 [hep-ph]].

[2] G. Degrassi, S. Di Vita, J. Elias-Miro, J. R. Espinosa, G. F. Giudice, G. Isidori and A. Strumia, Higgs mass and vacuum stability in the Standard Model at NNLO, JHEP 08 (2012), 098 [arXiv:1205.6497 [hep-ph]].

[3] P. Marquard, A. V. Smirnov, V. A. Smirnov and M. Steinhauser, Quark Mass Relations to FourLoop Order in Perturbative QCD, Phys. Rev. Lett. 114 (2015), 142002 [arXiv:1502.01030 [hep-ph]].

[4] P. Nason, The Top Mass in Hadronic Collisions, [arXiv:1712.02796 [hep-ph]].

[5] A. H. Hoang, What is the Top Quark Mass?, Ann. Rev. of Nucl. and Part. Sci. 70 (2020), [arXiv:2004.12915 [hep-ph]].

[6] ATLAS Collaboration, The ATLAS Experiment at the CERN Large Hadron Collider, 2008 JINST 3 S08003.

[7] CMS Collaboration, The CMS experiment at the CERN LHC, 2008 JINST 3 S08004.

[8] ATLAS Collaboration, Measurement of the top quark mass using a leptonic invariant mass in pp collisions at $\sqrt{s}=13 \mathrm{TeV}$ with the ATLAS detector, ATLAS-CONF-2019-046, https://cds.cern.ch/record/2693954.

[9] CMS Collaboration, Measurement of the top quark mass in events with a single reconstructed top quark at $\sqrt{s}=13 \mathrm{TeV}$, CMS-PAS-TOP-19-009, https://cds.cern.ch/record/2759301.

[10] CMS Collaboration, Running of the top quark mass from proton-proton collisions at $\sqrt{s}=13$ TeV, Phys. Lett. B 803 (2020), 135263 [arXiv:1909.09193 [hep-ex]].

[11] J. M. Campbell and R. K. Ellis, MCFM for the Tevatron and the LHC, Nucl. Phys. B Proc. Suppl. 205-206 (2010), 10 [arXiv:1007.3492 [hep-ph]].

[12] B. Schmidt and M. Steinhauser, CRunDec: a C++ package for running and decoupling of the strong coupling and quark masses, Comput. Phys. Commun. 183 (2012), 1845 [arXiv:1201.6149 [hep-ph]].

[13] ATLAS Collaboration, A precise interpretation for the top quark mass parameter in ATLAS Monte Carlo simulation, ATL-PHYS-PUB-2021-034, https://cds.cern.ch/record/2777332.

[14] A. H. Hoang, A. Jain, I. Scimemi and I. W. Stewart, Infrared Renormalization Group Flow for Heavy Quark Masses, Phys. Rev. Lett. 101 (2008), 151602 [arXiv:0803.4214 [hep-ph]].

[15] A. J. Larkoski, S. Marzani, G. Soyez and J. Thaler, Soft Drop, JHEP 05 (2014), 146 [arXiv:1402.2657 [hep-ph]]. 
[16] M. Aliev, H. Lacker, U. Langenfeld, S. Moch, P. Uwer and M. Wiedermann, HATHOR: HAdronic Top and Heavy quarks crOss section calculatoR, Comput. Phys. Commun. 182 (2011), 1034-1046 [arXiv:1007.1327 [hep-ph]].

[17] CMS Collaboration, Measurement of the top quark Yukawa coupling from $\mathrm{t} \overline{\mathrm{t}}$ kinematic distributions in the dilepton final state in proton-proton collisions at $\sqrt{s}=13 \mathrm{TeV}$, Phys. Rev. D 102 (2020) no.9, 092013 [arXiv:2009.07123 [hep-ex]]. 